\title{
Feasibility analysis of treating severe intrauterine adhesions by transplanting menstrual blood-derived stem cells
}

\author{
SHENG-XIA ZHENG ${ }^{1}$, JIAN WANG ${ }^{2}$, XUE-LI WANG ${ }^{1}$, ASIM ALI $^{3}$, LI-MIN WU $^{1}$ and YU-SHENG LIU ${ }^{1}$ \\ ${ }^{1}$ Department of Reproductive Medicine; ${ }^{2}$ Hematology Laboratory, Anhui Provincial Hospital, \\ The First Affiliated Hospital of University of Science and Technology of China; ${ }^{3}$ Molecular and Cell Genetics Laboratory, \\ School of Life Sciences, University of Science and Technology of China, Hefei, Anhui 230001, P.R. China
}

Received October 8, 2016; Accepted January 9, 2018

DOI: $10.3892 /$ ijmm.2018.3415

\begin{abstract}
Intrauterine adhesions (IUA) are associated with the loss of stem cells in the endometrium. Menstrual blood-derived stem cells (MenSCs) can be isolated from the menstrual blood and differentiated into endometrial cells. To check the transplantation feasibility of MenSCs for the treatment of severe IUA, MenSCs were isolated from menstrual blood, cultured in Dulbecco's modified Eagle's medium (DMEM), identified by immunocytochemistry and flow cytometry, differentiated into endometrial cells in vitro, and finally transplanted into the axillary subcutaneous tissue of non-obese diabetic/severe combined immunodeficiency (NOD-SCID) mice to create endometrial tissue. Additionally, the cloning efficiency and POU domain class 5 transcription factor 1 (OCT-4) positivity of MenSCs from patients with severe IUA were compared with those from healthy women. Immunocytochemistry and flow cytometry results showed that $95.1 \pm 0.8 \%$ cells were OCT-4-positive, $0.9 \pm 0.4 \%$ were cluster of differentiation (CD) 45 -positive, $1.8 \pm 0.9 \%$ were STRO-1-positive and $1.0 \pm 0.4 \%$ were human leukocyte antigen-antigen D related-positive. Following differentiation in vitro, the results of immunocytochemistry, reverse transcription-polymerase chain reaction and western blot analysis showed that the expression of cytokeratin (CK) and vimentin (VIM) was increased in MenSCs compared with that in control subjects. Subsequent to transplantation in mice administered with sequential $17 \beta$-estradiol and progesterone, CK, VIM, estrogen receptor and progesterone receptor were expressed in the transplantation regions, suggesting that MenSCs could differentiate into endometrial tissues in vivo. The cloning efficiency and OCT-4 positivity of MenSCs from patients with
\end{abstract}

Correspondence to: Professor Yu-Sheng Liu, Department of Reproductive Medicine, Anhui Provincial Hospital, The First Affiliated Hospital of University of Science and Technology of China, 17 Lujiang Road, Hefei, Anhui 230001, P.R. China

E-mail: shengzhiliuvip@163.com

Key words: menstrual blood-derived stem cells, endometrial stem cells, culture, transplantation, intrauterine adhesions severe IUA was significantly decreased. In conclusion, to the best of our knowledge, this is the first study in which MenSCs could differentiate into endometrial cells in vitro and create endometrial tissue in NOD-SCID mice in vivo, with impaired cloning efficiency and OCT-4 expression of MenSCs from patients with IUA. This study will provide a theoretical basis for the treatment of IUA with MenSCs.

\section{Introduction}

Since the first embryonic stem cells (ESCs) were established from mice in 1981 (1), research on stem cells has made great progress. Due to their abilities of multi-directional differentiation, self-renewal and paracrine functions, stem cells show enormous potential in cell therapy (2) for a number of conditions, including neurodegenerative diseases, bone damage, Duchene muscular dystrophy and diabetes (3-5). However, the ethical issues, possible tumorigenicity and risk of differentiated tissue rejection currently limit their use in clinical practice.

Mesenchymal stem cells (MSCs) are currently the most 'popular' stem cells; they not only have high self-renewal and multi-lineage differentiation potential (6), but also have the ability to migrate to damaged tissues and areas with chronic inflammation to regulate the immune response $(7,8)$. MSCs from the bone marrow (BMSCs) (9), cord blood (10), adipose tissue (11) and amniotic fluid (12) have been studied for the treatment of various illnesses (13), including bone defects (14), cartilage defects $(15,16)$, myocardial infarction (17), cerebral ischemia (18), lung injury (19), liver damage (20), limb ischemia (21) and skin damage (22).

Intrauterine adhesions (IUAs) are consistent with the symptoms of pain, menstrual disturbances, amenorrhea and infertility. Due to the inadequate thickness of the uterine wall (endometrium), it is difficult to conceive naturally or even carry an assisted pregnancy to term (23). In IUAs, fibrotic scar tissue may partially or completely fill the uterine cavity (24). The loss of endometrial stem cells directly causes fibrous tissue proliferation and subsequent synechiae (25).

There is little research in the literature about how to treat IUAs using BMSCs. BMSCs were previously reported to treat endometrial lesions and IUAs successfully in mice (26-28). 
The treatment of patients with IUAs with autologous BMSC transplantation is beneficial for the regeneration of an injured endometrium (29). Furthermore, it was reported in 2011 that a patient with IUAs conceived and completed a successful pregnancy following autologous BMSC transplantation treatment (30).

However, different factors, including age, invasive acquisition and type of disease, restrict the proliferation ability and the efficacy of BMSCs. In addition, although the puncture and extraction of bone marrow is relatively safe, there is a risk of hemorrhage and infection. Ideal stem cells should have a high proliferation ability, multi-lineage differentiation potential, no immunogenicity, ease of collection and no ethical controversy (31). In recent years, endometrial stem cells (EnSCs) from the menstrual blood-derived stem cells (MenSCs) have gradually attracted the attention of a number of researchers $(13,31,32)$.

The use of MenSCs has numerous advantages in the treatment of IUAs. First, these stem cells can be obtained easily and non-invasively. Second, MenSCs have the qualities of widespread expansion and rapid amplification. Third, MenSCs exhibit higher expression of matrix metalloproteases. Physiologically, it is known that regularly remodeling of endometrial tissue is associated with the process of menstruation; thus, the potential role of these cells in remodeling the endometrium has been investigated (13).

However, much research is required to assess the capability of MenSCs as a potential therapy for the treatment of women with reproductive issues. Therefore, the present study aimed to provide a basis of treatment by the use of MenSCs in patients with severe IUAs. MenSCs were cultured, identified and induced toward endometrial cells, and their potential in creating endometrial tissues was assessed in mice. In addition, MenSCs from women with severe IUAs were compared with those of healthy women with a normal uterine cavity. Of course, for patients who do not menstruate, it is not possible to collect menstrual blood. Thus, these women are not within the scope of the present discussion.

\section{Materials and methods}

Animals. A total of 10 female non-obese diabetic/severe combined immunodeficiency (NOD-SCID) mice (aged 6-8 weeks and weighing 20-22 g) were purchased from the National Rodent Laboratory Animal Center (Shanghai, China) for this study and maintained according to the Institutional Animal Care and Use Committee of the Anhui Medical University (Hefei, Anhui, China). Animals were raised under specific pathogen-free conditions with a 12/12-h day/night schedule. The temperature was kept at $16-20^{\circ} \mathrm{C}$, and the humidity was maintained at $50-60 \%$. Animals had free access to food and water. This study followed the 1996 National Institutes of Health Guide for Experimental Animal Protection and Application. In this study, MenSCs were transplanted into axillary subcutaneous tissue, and the transplanted subcutaneous tissue was later removed under local anesthesia; thus, no mice were euthanized. The use of experimental animals was approved by the Experimental Animal Management Committee of Provincial Hospital Affiliated with Anhui Medical University.
Specimens. Menstrual blood and endometrium specimens were obtained from 5 patients with infertility due to IUAs and 5 healthy female volunteers who had a history of term delivery and a normal menstrual quantity. Subjects were from the Reproductive Medical Center of Anhui Provincial Hospital (Hefei, Anhui, China). Selection criteria were as follows: i) Age of 24-38 years; ii) no hormone therapy within 3 months of sample collection; iii) no irregular uterine bleeding; iv) no endometriosis; and v) no polycystic ovarian syndrome; and vi) no hormone-dependent disease of any type. The study was approved by the Ethics Committee of the Anhui Provincial Hospital Affiliated with Anhui Medical University. All patients who agreed to participate in this study provided written informed consent, and the human samples were anonymized prior to use. Medical and human waste were disposed in accordance with the national standard.

Culture and phenotypic characterization of MenSCs. MenSCs were isolated as described previously (33). Briefly, menstrual blood $(5 \mathrm{ml})$ from the second day of normal menstrual period was collected with a menstrual cup and transferred into phosphate-buffered saline (PBS) containing $0.2 \mathrm{ml}$ amphotericin B, $0.2 \mathrm{ml} 1 \%$ penicillin and streptomycin, and $0.1 \mathrm{ml}$ ethylenediamine tetraacetic acid-disodium. The menstrual blood was diluted using the same volume of aseptic PBS. MenSCs were separated by $1.077 \mathrm{~g} / \mathrm{ml}$ lymphocyte separation medium (Institute of Hematology, Chinese Academy of Medical Sciences, Tianjin, China) with a density gradient centrifugation of $400 \mathrm{x} \mathrm{g}$ for $25 \mathrm{~min}$ at room temperature. The cells were inoculated in low-glucose Dulbecco's modified Eagle's medium (DMEM; HyClone; GE Healthcare Life Sciences, Logan, UT, USA) (34) containing $10 \%$ fetal bovine serum (FBS; Gibco; Thermo Fisher Scientific, Inc., Chicago, IL, USA) and cultured at $37^{\circ} \mathrm{C}$ with $5 \% \mathrm{CO}_{2}$ and saturated humidity. The medium was changed every 3 to 4 days according to the growth of the cells. MenSCs were characterized on the basis of their phenotype by flow cytometry and immunocytochemistry.

Flow cytometry. Following digestion and washing, $5 \times 10^{5}$ MenSCs detached from culture bottles were incubated with $1 \%$ bovine serum albumin (BSA)/PBS (Gibco) at room temperature for $30 \mathrm{~min}$ to block non-specific antigens. $50 \mu \mathrm{l}$ cell suspension $\left(\sim 5 \times 10^{5}\right.$ cells) was transferred into two test tubes, labeled as 1 and 2, respectively. A total of $3 \mu$ fluorescein isothiocynanate (FITC)-conjugated anti-STRO-1 ( $2 \mathrm{mg} / \mathrm{ml} ; 1: 100$ dilution; BD Biosciences, Franklin Lakes, NJ, USA) and $4 \mu 1$ allophycocyanin (APC)-conjugated anti-cluster of differentiation (CD) $45(1 \mathrm{mg} / \mathrm{ml} ; 1: 100$ dilution; 103111; BioLegend, San Diego, CA, USA) were added to the test tube labeled 1 , and $3 \mu 1$ phycoerythrin (PE)-conjugated anti-human leukocyte antigen-antigen D related (HLA-DR; $2 \mathrm{mg} / \mathrm{ml} ; 1: 100$ dilution; cat. no. MAB4869; Bio-gems, Westlake Village, CA, USA) was added to the test tube labeled 2. The test tubes were incubated for $15 \mathrm{~min}$ at room temperature in the dark. Subsequent to fixation for $15 \mathrm{~min}$ and washing with PBS, permeabilization agents were added into test tube 1 for $5 \mathrm{~min}$, and then $2.5 \mu \mathrm{l}$ PE-conjugated anti-POU domain class 5 transcription factor 1 (OCT-4-PE; $2 \mathrm{mg} / \mathrm{ml} ; 1: 100$ dilution; cat. no. 560186; BD Biosciences) was added to the tube and 
incubated for $15 \mathrm{~min}$ at room temperature. All antibodies were added according to the manufacturers' recommended concentrations and methods, simultaneously setting blank and isotype-matched controls. Tubes were then resuspended in $5 \%$ fetal calf serum (FCS)/PBS containing propidium iodide (10 mg/ml; Sigma-Aldrich; Merck KGaA, Darmstadt, Germany) and fluorescence-activated cell sorting (FACS) on the FACSCalibur (BD Canto II; BD Biosciences) using FlowJo software (version 7.6.1; Cytomation Inc., Stanford University, Carpinteria, CA, USA). The majority of cells were selected for analysis based on the forward versus side scatter profile. Dead cells were excluded by electronic gating, and remaining cells were analyzed for STRO-1-FITC, CD45-APC, HLA-DR-PE and OCT-4-PE.

To detect the proportions of the different stem cells or progenitor cells among MenSCs, epithelial cell adhesion molecule/CD326-PerCP-Cy5-5 (epithelial progenitor cells; 1:100 dilution, cat. no. 563180; BD Biosciences), platelet endothelial cell adhesion molecule/CD31-PE-Cy7 (endothelial progenitor cells; 1:100 dilution, cat. no. 558094; BD Biosciences) and melanoma cell adhesion molecule/CD146-APC (ectomesenchymal stem cells; 1:100 dilution; cat. no. 562231; BD Biosciences) marker antibodies were used in combination with OCT-4-PE antibody to label the MenSCs, and FACS determination of OCT-4CD326, OCT-4CD31 and OCT-4CD146 populations was performed. Finally, the analysis was performed using FlowJo software.

Immunocytochemistry. For immunocytochemistry, subsequent to washing with PBS, the cells grown on cover slips were fixed in $95 \%$ ethanol for $15 \mathrm{~min}$ at room temperature. Immunocytochemistry was performed as follows: Endogenous peroxidase activity was blocked using a solution of $0.15 \% \mathrm{H}_{2} \mathrm{O}_{2}$ in methanol prior to permeabilizing the cells in $0.2 \%$ IGEPAL, $1 \%$ BSA (both Sigma-Aldrich; Merck $\mathrm{KGaA}$ ) and $10 \%$ normal goat serum (NGS; Biosera, Labtech International, Ltd., East Sussex, UK). Non-specific binding was blocked using $20 \% \mathrm{NGS} / 5 \%$ BSA prior to endogenous strepavidin and biotin being blocked according to the manufacturer's protocols (Vector Laboratoris, Burlingame, CA, USA). The cells were incubated with primary antibody against OCT-4 (cat. no. ab184665), STRO-1 (cat. no. ab214086), CD45, HLA-DR (cat. no. ab136320) (all 1:100 dilution; Abcam, Cambridge, MA, USA), cytokeratin (CK; 1:100 dilution; ZSJQ, ZM-0067) and vimentin (VIM) (1:100 dilution; ZSJQ, ZA-0511) overnight at $4^{\circ} \mathrm{C}$. The secondary antibody, streptavidin peroxidase (1:100 dilution; Wuhan Boster Biological Technology, Ltd., Wuhan, China) was added for $30 \mathrm{~min}$ at $37^{\circ} \mathrm{C}$. Following reaction with $0.01 \%$ 3,3'-diaminobenzidine (BioGenex, Fremont, CA, USA) for $5 \mathrm{~min}$, cells were dyed with hematoxylin and dehydrated in $95 \%$ ethanol. Slides were evaluated independently by three pathologists under an inverted microscope (Olympus, Tokyo, Japan). The pathologists were blinded to the origin of the samples.

Karyotypic analysis. For karyotypic analysis, the cultured MenSCs were doubled 36 times and harvested at 70-80\% confluency; the MenSCs were resuspended in $10 \mu \mathrm{l}$ of colcemid per $\mathrm{ml}$ of media. Cells were incubated at $37^{\circ} \mathrm{C}$ for 3-6 $\mathrm{h}$ and then resuspended in $0.5 \mathrm{ml}$ medium and mixed
Table I. Primers for reverse transcription-quantitative polymerase chain reaction.

\begin{tabular}{lcc}
\hline Primer & Sequence & Bp \\
\hline GAPDH & & 158 \\
Forward & 5'-GTCAAGGCTGAGAACGGGAA-3' & \\
Reverse & 5'-AAATGAGCCCCAGCCTTCTC-3' & \\
CK8 & & 198 \\
Forward & 5'-TCATACGAAGACCACCAGCG-3' & \\
Reverse & 5'-GACTCAGACACCAGCTTCCC-3' & \\
VIM & & 198 \\
Forward & 5'-GGAGTCCACTGAGTACCGGA-3' & \\
Reverse & 5'-AGGTGACGAGCCATTTCCTC-3' & \\
\hline
\end{tabular}

GAPDH, glyceraldehyde 3-phosphate dehydrogenase; CK, cytokeratin; VIM, vimentin.

with $0.075 \mathrm{M} \mathrm{KCl}$ to a volume of $10 \mathrm{ml}$. Following incubation for $10-15 \mathrm{~min}$ at $37^{\circ} \mathrm{C}$ in a water bath, the cells were resuspended in a total of $10 \mathrm{ml}$ fixative (methonal:acetic acid at 3:1). Staining with 4',6-diamidino-2-phenylindole for G-banding was performed by equilibrating the slides in $0.3 \mathrm{M}$ sodium citrate containing $3 \mathrm{M} \mathrm{NaCl}$ for $5 \mathrm{~min}$, and then the cells were visualized under the microscope.

Proliferation and differentiation of MenSCs. MenSCs were proliferated into a single-cell suspension $\left(2 \times 10^{7}\right)$ in low-glucose DMEM containing $10 \%$ FBS, $1 \%$ amphotericin B and $1 \%$ penicillin/streptomycin. Each well of a 96 -well plate was filled with $100 \mu \mathrm{l}$ cell suspension. The plate was incubated at $37^{\circ} \mathrm{C}$ with $5 \% \mathrm{CO}_{2}$ and saturated humidity. Subsequent to $1,3,5,7,9$ and 14 days of culture, $10 \mu \mathrm{l}$ Cell Counting kit-8 (CCK-8; Beyotime Institute of Biotechnology, Chengdu, China) was added and incubated for $2 \mathrm{~h}$. The optical density at $450 \mathrm{~nm}$ was detected by spectroscopy (RT-6000; Nanjing Microscope Co., LTD, Nanjing, China), and the growth curve was drawn.

MenSCs $\left(5 \times 10^{4} / \mathrm{ml}\right)$ at the third passage were placed in 24-well plates with low-glucose DMEM containing 5\% FBS. Cells were divided into two groups. The control group was not exposed to any hormones or cytokines. The experimental group of cells were incubated with $10 \mathrm{ng} / \mathrm{ml}$ transforming growth factor- $\beta$ (TGF- $\beta$ ), $10 \mathrm{ng} / \mathrm{ml}$ epidermal growth factor (EGF), $10 \mathrm{ng} / \mathrm{ml}$ platelet-derived growth factor BB (PDGF-BB) (all Peprotech, Inc., Rocky Hill, NJ, USA) and $1 \times 10^{-8} \mathrm{~mol} / 1$ $17 \beta$-estradiol valerate (Sigma-Aldrich) (35). Subsequent to 5 days of induced differentiation at $37^{\circ} \mathrm{C}$, with $5 \% \mathrm{CO}_{2}$ and saturated humidity, the changes in cell morphology were observed and the expression of CK for endometrial epithelial cells and VIM for endometrial stromal cells were detected using immunocytochemistry.

Reverse transcription-quantitative polymerase chain reaction (RT-qPCR). The total RNA of induced MenSCs was isolated by TRIzol (Invitrogen; Thermo Fisher Scientific, Inc.), and the purity and concentration of RNA were measured by ultraviolet 
spectrometry. Single-stranded cDNA was synthesized using $2 \mu \mathrm{g}$ RNA and $5 \mathrm{X}$ reaction buffer (Takara Bio,Inc., Otsu, Japan), Ribolock and dNTP mix. The primers are shown in Table I (Sangon Biotech, Co., Ltd., Shanghai, China). Glyceraldehyde 3-phosphate dehydrogenase (GAPDH) was used as the internal control. RT-qPCR using the ABI Prism 7000 Sequence Detection system (Applied Biosystems, Foster City, CA, USA) was performed with $20 \mu$ l of the single-stranded cDNA sample with SYBR-Green PCR master mix (Takara) following the manufacturer's protocol. Melting curve analysis was performed subsequent to the RT-qPCR to monitor PCR specificity. Reactions were performed as follows: Denaturation at $93^{\circ} \mathrm{C}$ for $3 \mathrm{~min}$, then 40 cycles at $93^{\circ} \mathrm{C}$ for $45 \mathrm{sec}$ and $55^{\circ} \mathrm{C}$ for $20 \mathrm{sec}$. All experiments were repeated three times. The $2^{-\Delta \Delta \mathrm{Cq}}$ method (36) was used to represent the relative expression of target gene mRNA.

Western blot analysis. Induced MenSCs were lysed with lysis buffer containing the phenylmethylsulfonyl fluoride protease inhibitor (Beyotime Institute of Biotechnology). Cells were centrifuged at $20,000 \times \mathrm{g}$ at $4^{\circ} \mathrm{C}$ for $12 \mathrm{~min}$. The supernatant was obtained. Protein $(25 \mathrm{ng})$, as quantified using a bicinchoninic acid protein assay kit (Thermo Fisher Scientific, Inc.), was separated by $12 \%$ sodium dodecyl sulfate-polyacrylamide gel electrophoresis. Following electrophoresis, the proteins were transferred to polyvinylidene fluoride membranes. The membranes were incubated in blocking solution containing 5\% non-fat milk for $1.5 \mathrm{~h}$ at room temperature and incubated with the corresponding primary antibodies [CK (cat. no. TA803188), VIM (cat. no. TA801319) and GAPDH (cat. no. TA802563S), all 1:100 dilution; OriGene Technologies, Inc.] overnight at $4^{\circ} \mathrm{C}$. Subsequent to being washed three times, the blots were incubated with the appropriate horseradish peroxidase-conjugated secondary antibody (Cell Signaling Technology, Danvers, MA, USA) [1:1,000 dilution in Tris-buffered saline with Tween (TBST) buffer] for $1 \mathrm{~h}$ at room temperature. Proteins were visualized using an enhanced chemiluminescence kit [diaminobenzidine (DAB); Biyuntian Company, Shanghai, China]. The densities of bands were analyzed by a gel imaging system and calculated compared to the internal control.

Endometrium rebuilt in vivo in mice. A total of $1.46 \mu \mathrm{g}$ gonadotropin-releasing hormone agonist (Ipsen Pharma Biotech, Signes, France) was administered to each of the 10 female NOD-SCID mice every 2 days via intramuscular injection for downregulation pituitary. At 4 days post-injection, $1 \times 10^{6}$ MenSCs of the forth passage detached from culture bottle in $30 \mu \mathrm{l}$ DMEM were transplanted into one side of the axillary subcutaneous tissue, and the same volume of DMEM was transplanted into the other side of the axillary subcutaneous tissue as a control. Mice were treated with $3 \mu \mathrm{g} /$ day $17 \beta$-estradiol valerate (Sigma-Aldrich; Merck KGaA) for 4-6 weeks. Transplanted subcutaneous tissue was removed under local anesthesia in 5 mice. The remaining 5 mice received progesterone $1 \mathrm{mg} /$ day for 2 weeks. The resected tissue was fixed with $10 \%$ formaldehyde at room temperature overnight and embedded in paraffin and cut into 5- $\mu \mathrm{m}$ sections. The expression of CK, VIM, CD31 (cat. no. TA504964) (1:100 dilution; all OriGene Technologies, Inc., Rockville, MD,
USA), estrogen receptor (ER) (1:100 dilution; cat. no. ab17230; Abcam, Hong Kong, China) and PR (Yongyi Biotechnology, Co., Shanghai, China) was detected by immunohistochemistry.

Immunohistochemistry. Immunohistochemistry staining was performed to detect the expression of OCT-4, CD146, CD31 and CD326 in $5-\mathrm{mm}^{3}$ of endometrial IUA (diagnosed by hysteroscopy) and endometrium of a normal uterine cavity, as well as CK, VIM, CD31, ER and PR in a rebuilt endometrium in NOD-SCID mice. Immunohistochemical staining was performed on formalin-fixed, paraffin-embedded sections using the labeled horseradish peroxidase (HRP) method. The series of paraffin sections, $5-\mu \mathrm{m}$ thick, were mounted on 3-aminopropyltriethoxy-silane-coated slides, deparaffinized and rehydrated in a gradient alcohol series and distilled water. The sections were heated in citrate buffer $(\mathrm{pH} \mathrm{6.0;}$ Sigma-Aldrich; Merck KGaA) in a microwave oven for $20 \mathrm{~min}$ for antigen retrieval. The sections were then cooled naturally to room temperature, and washed in TBS, then TBST, respectively, for $5 \mathrm{~min}$. The sections were immersed in 3\% BSA (FBS; Gibco; Thermo Fisher Scientific, Inc.) for $15 \mathrm{~min}, 10 \%$ donkey serum (ab7475; Abcam) and $0.1 \%$ Triton X-100 (BDT) block solution for $30 \mathrm{~min}$ at $37^{\circ} \mathrm{C}$, and incubated with monoclonal antibody against OCT-4 (1:100 dilution; cat. no. RS-1111R), CD146 (1:100 dilution; cat. no. BA3996-2), CD31 (1:100 dilution; cat. no. SV000-01), CD326 (1:100 dilution; cat. no. RS-4889R) (all Abcam), CK (1:100 dilution), VIM (1:100 dilution), ER (1:100 dilution) and PR (1:100 dilution) (all OriGene Technologies, Inc.) at $4^{\circ} \mathrm{C}$ overnight. Negative controls were incubated with antibody diluent only. The sections were then thoroughly washed with $0.1 \%$ Tween-20 in TBS three times and incubated with Envision ${ }^{\mathrm{TM}}$ HRP-conjugated secondary antibody (1:100 dilution; cat. no. K5007; Dako; Agilent Technologies, Inc., Santa Clara, CA, USA) for $1 \mathrm{~h}$ at room temperature, followed by washing three times with $0.1 \%$ Tween-20 TBS. Counterstaining was performed with hematoxylin at room temperature for a few minutes and visualized under a light microscope.

Cloning efficiency of MenSCs in severe IUA and normal endometrium. MenSCs were separated from the menstrual blood of patients with severe IUA (diagnosed by hysteroscopy) (IUA group) and from healthy women with a normal endometrium (control group). Cell culture inoculation steps were followed as previously stated for the MenSCs. Following 15 days of primary culture, the cloning efficiency of the MenSCs was assessed and calculated as follows: Cloning efficiency $(\%)=$ clones $/$ cells $\mathrm{x} 100$.

Statistical analysis. All results were performed in triplicate. The data for the immunocytochemistry and immunohistochemistry were from at least 5 randomly selected sections. All sections were observed by 2 authoritative pathologists using a light microscope. Continuous data are expressed as the mean \pm standard error of the mean and were analyzed by Student's t-test. Analyses were performed using SPSS 17.0 (SPSS, Inc., Chicago, IL, USA). Two-sided P-values of $<0.05$ were considered to indicate a statistically significant difference. 
A

$24 \mathrm{~h}$

B

$72 \mathrm{~h}$
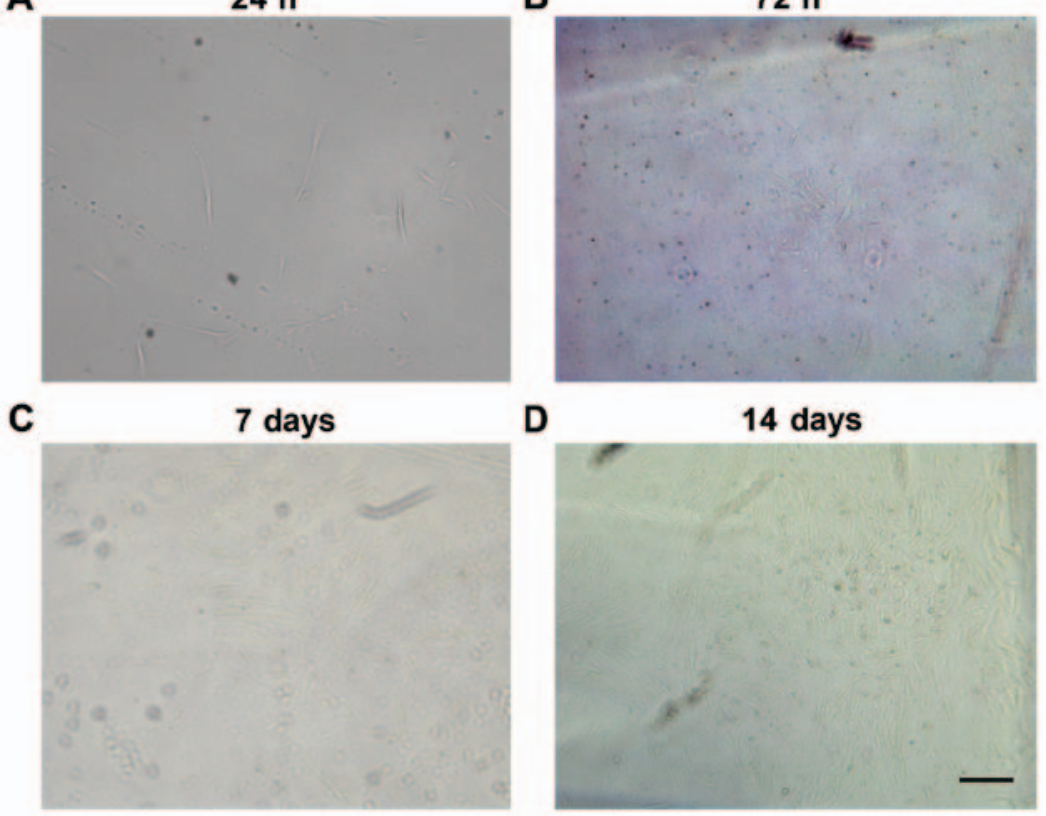

Figure 1. Morphology and growth characteristics of normal menstrual blood-derived stem cells. Subsequent to (A) 24 h, (B) 72 h, (C) 7 days and (D) 14 days of culture. Scale bar, $50 \mu \mathrm{m}$.

\section{Results}

Morphology and cloning of MenSCs. Following $24 \mathrm{~h}$ of primary culture, there was a small amount of spindle- and polygonal-shaped adherent cells mixed with circular blood cells. Subsequent to removing the non-adherent cells at $72 \mathrm{~h}$, the adherent cells were well distributed on the plate, and displayed a spindle-like morphological appearance and irregular triangular fibers. It was observed that dozens of cells formed small clones, and the distribution was heterogeneous. Subsequent to 6-7 days, small clones grew into large clones with hundreds of cells. Cells had spindle-like, irregular triangular, cup shapes, with long protrusions and high refraction. When the culture was continued to 12-14 days, adherent cells quickly proliferated. Cells were arranged in single swirl, radial or fence-like patterns. The main morphology of the cells was spindle-like (Fig. 1). With the increase of passages, cells with irregular triangular and cup shapes were eliminated due to poor development. These may have been no stem cell properties of terminal differentiation cells.

Proliferation and characteristics of MenSCs. The shape of the cells did not change with the increase of passage. Karyotyping showed that 41 metaphases were imaged with 38 cells having G-banding following 36 doublings. This showed a normal karyotype of 46 chromosomes, 23 pairs, with no aneuploidy, tetraploidy or other visible abnormalities (Fig. 2A), which suggests that the MenSCs could show large-scale expansion without mutagenesis, at least at the chromosomal level.

To detect whether the cultured MenSCs were stem cells, immunocytochemistry was used to label the stem cell or progenitor cell markers. Hematoxylin and eosin (H\&E) staining showed that the nuclei and cytoplasm were lightly dyed, and the cytoplasm exhibited an irregular shape. Overall, $>90 \%$ of the MenSCs were positive for OCT- 4 (embryonic stem cell marker) and negative for the expression of STRO-1 (ectomesenchymal stem cells marker), CD45 (hematopoietic stem cell marker) and HLA-DR (human leukocyte antigen marker). Approximately $10 \%$ of nuclei were OCT-4-positive (Fig. 3A). These results suggested that the cultured MenSCs were mostly OCT-4-positive stem cells.

To further confirm that the cultured MenSCs were stem cells, OCT-4, STRO-1, CD45 and HLA-DR antibodies were used to label the cells for flow cytometry. The cultured cells were primarily OCT-4-positive stem cells with low immunogenicity. The exact profile was $95.1 \pm 0.8 \%$ positive for OCT- 4 , $1.8 \pm 0.9 \%$ positive for STRO- $1,0.9 \pm 0.4 \%$ positive for CD 45 and $1.0 \pm 0.4 \%$ positive for HLA-DR (Fig. 3B).

As it is well-known that endometrial stem cells are heterogeneous, a detailed analysis showed that the dominant proportion of MenSCs were OCT $-4^{+} / \mathrm{CD}_{146}{ }^{+}$cells, suggesting that the isolated MenSCs were ectomesenchymal stem cells. Indeed, $33.5 \pm 1.5 \%$ were $\mathrm{CD} 326^{+}, 25.3 \pm 1.7 \%$ were $\mathrm{OCT}^{+} / \mathrm{CD} 326^{+}, 3.3 \pm 0.3 \%$ were $\mathrm{CD} 31^{+}, 2.3 \pm 0.2 \%$ were $\mathrm{OCT}^{+} / \mathrm{CD} 31^{+}, 78.0 \pm 1.6 \%$ were $\mathrm{CD} 146^{+}$ and $39.0 \pm 1.4 \%$ were $\mathrm{OCT}_{4}+\mathrm{CD}_{146}{ }^{+}$(Fig. $3 \mathrm{C}$ ).

MenSC differentiation into endometrial cells. Subsequent to 5 days of induced culture, MenSCs in the differentiation phase became smaller, with a decreased nucleus-to-cytoplasm ratio. The signal intensity of immunocytochemistry was scored as follows: 0 , negative (no immunopositivity); 1 , weak (low immunopositivity); 2 , moderate (moderate immunopositivity); and 3, strong (intense immunopositivity). CK and VIM expression in the control group was very weakly positive (score $=1$ ), whereas CK and VIM expression by immunocytochemistry was strongly positive (score $=3$ ) in the differentiation group, suggesting that MenSCs had differentiated into endometrial cells (Fig. 4A). RT-qPCR and western blot analysis showed that the expression of CK and VIM mRNA and protein was increased in the differentiation group compared with that in the control group $(\mathrm{P}<0.05)$ (Fig. 4B and $\mathrm{C})$. 

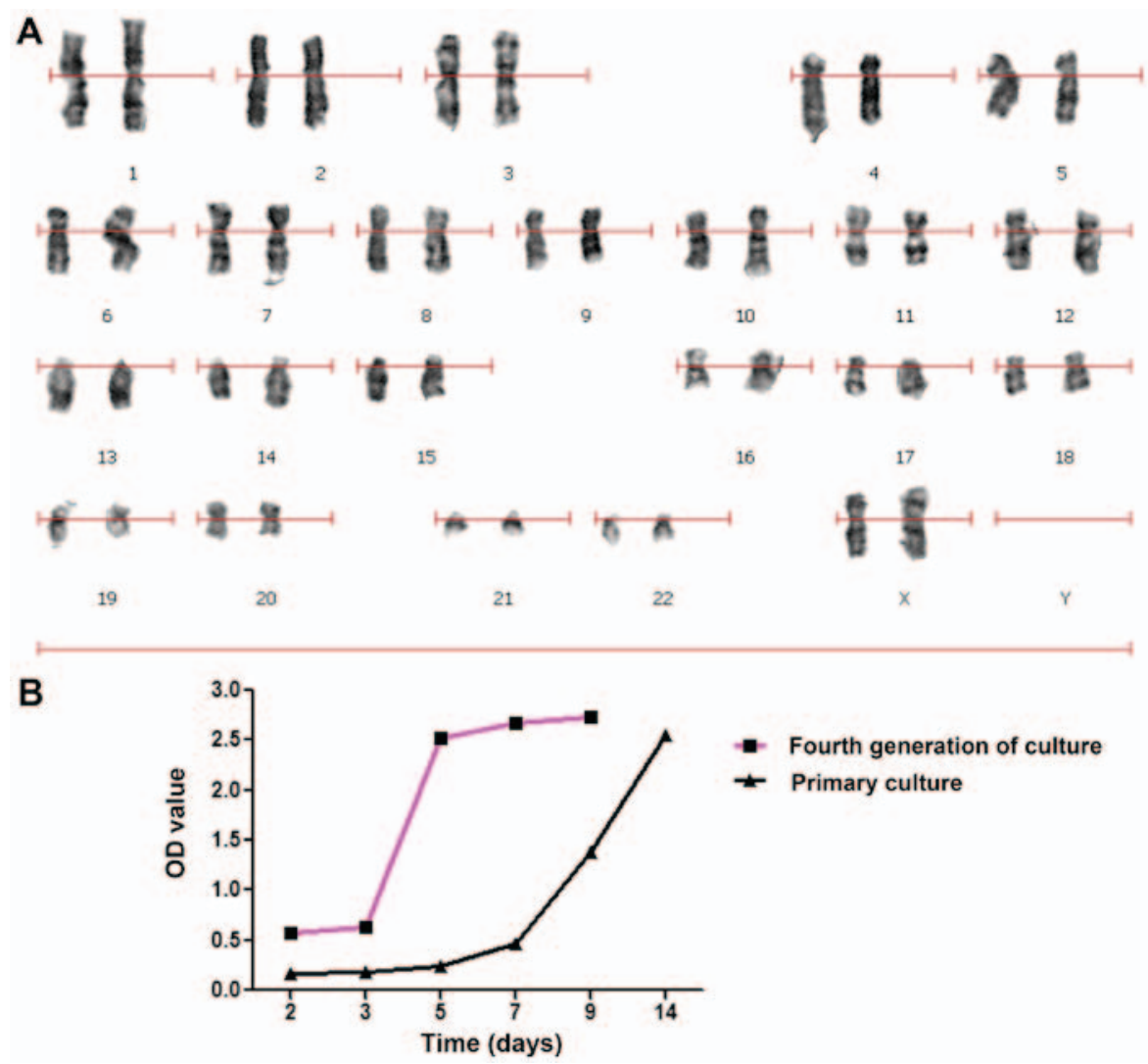

Figure 2. Karyotypic analysis and proliferation of normal MenSCs. (A) MenSCs after 36 doubling times were harvested for karyotype analysis. Cells were stained with 4',6-diamidino-2-phenylindole for G-banding. 46 chromosomes were normal. (B) The cell counting kit-8 method was used to assess the proliferation of the MenSCs. A growth curve was drawn according to the OD values in passage 0 and passage 4. MenSCs, menstrual blood-derived stem cells; OD, optical density.

A a

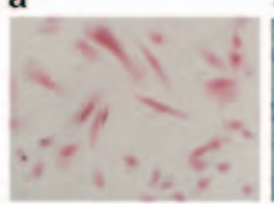

b
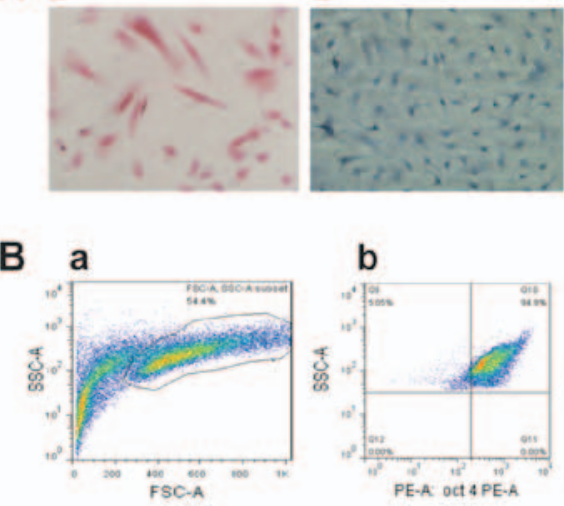

C a

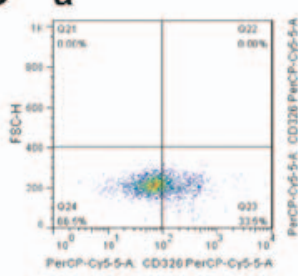

b

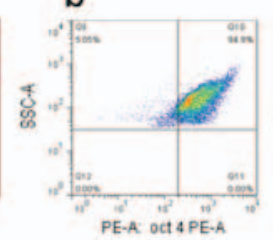

b

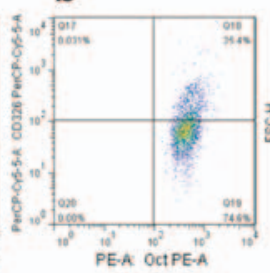

c

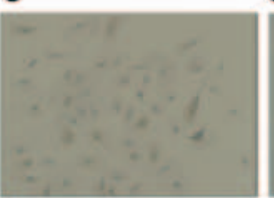

d

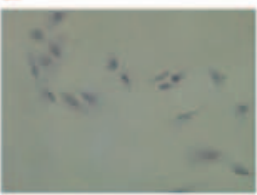

e

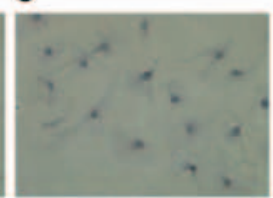

f

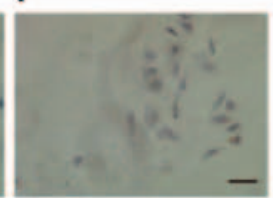

C

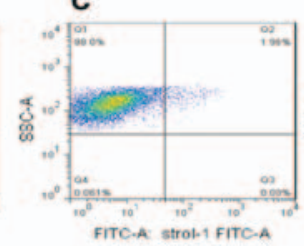

c

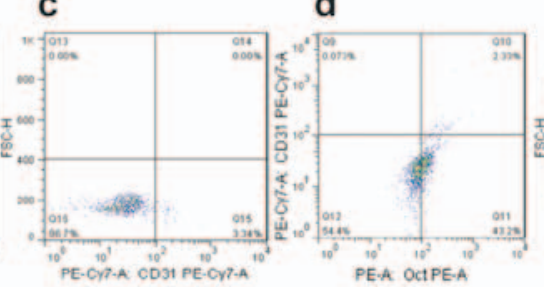

d

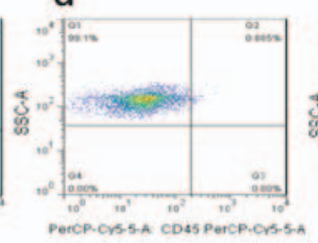

e

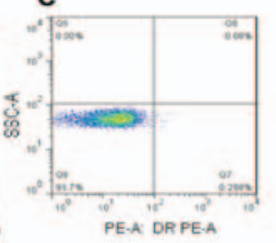

e $f$

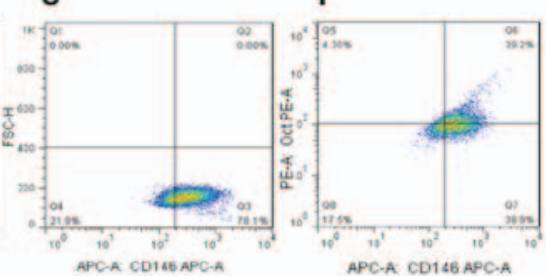

Figure 3. Expression of OCT-4, STRO-1, CD45 and HLA-DR were detected in cultured normal MenSCs. (A) Immunocytochemistry: (a) H\&E, (b) negative control for OCT-4; (c) OCT-4; (d) STRO-1; (e) CD45; and (f) HLA-DR (scale bar, $20 \mu \mathrm{m}$ ). (B) Detected proportion of positive cells occupy cultured MenSCs by flow cytometry: (a) Cell gating; (b) OCT-4, $95.1 \pm 0.8 \%$; (c) STRO-1, $1.8 \pm 0.9 \%$; (d) CD45, 0.9 $\pm 0.4 \%$; and (e) HLA-DR, 1.0 $\pm 0.4 \%$. (C) Detected heterogeneous population proportion in cultured MenSCs by flow cytometry of epithelial cell adhesion molecule/CD326, platelet endothelial cell adhesion molecule/CD31 and melanoma cell adhesion molecule/CD146 antibodies were combined with OCT-4 antibody: (a) CD326 $6^{+}, 33.5 \%$; (b) $\mathrm{CD}_{2} 26^{+} / \mathrm{OCT}^{-} 4^{+}, 25.3 \%$; (c) $\mathrm{CD} 31^{+}$, $3.31 \%$; (d) $\mathrm{CD} 31^{+} / \mathrm{OCT}-4^{+}, 2.32 \%$; (e) CD146 ${ }^{+}, 78.0 \%$; and (f) $\mathrm{CD} 146^{+} / \mathrm{OCT}-4^{+}, 39.0 \%$. OCT-4, POU domain class 5 transcription factor 1; HLA-DR, human leukocyte antigen-antigen D related; $\mathrm{CD}$, cluster of differentiation; MenSCs, menstrual blood-derived stem cells; PE, phycoerythrin; FITC, fluorescein isothiocyanate; SSC, side scatter; FSC, forward scatter. 
A a

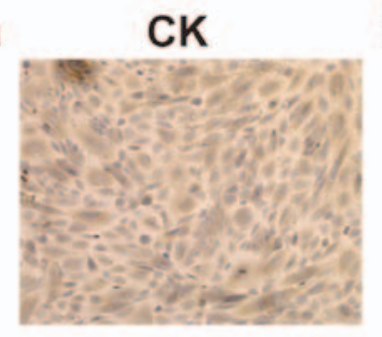

b VIM

C

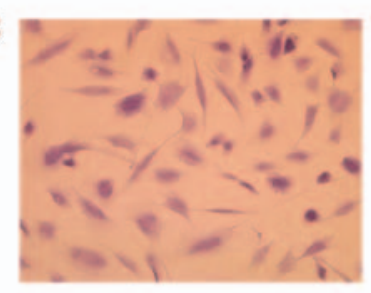

e

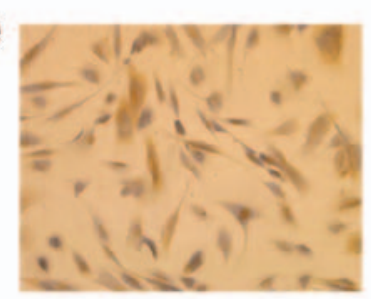

g
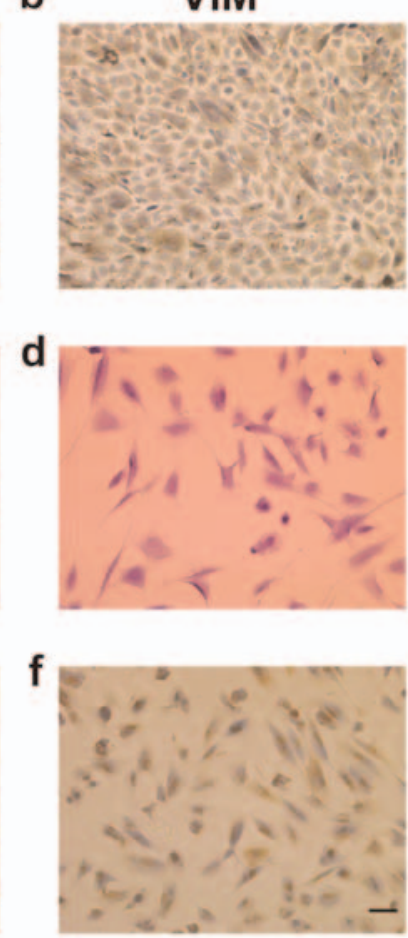

$\mathrm{DCK}$

$\mathrm{VIIM}$

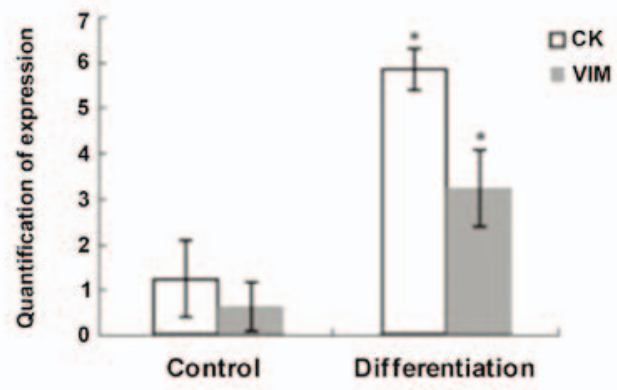

B

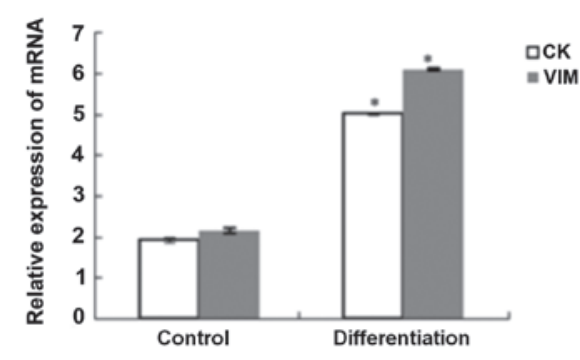

C a
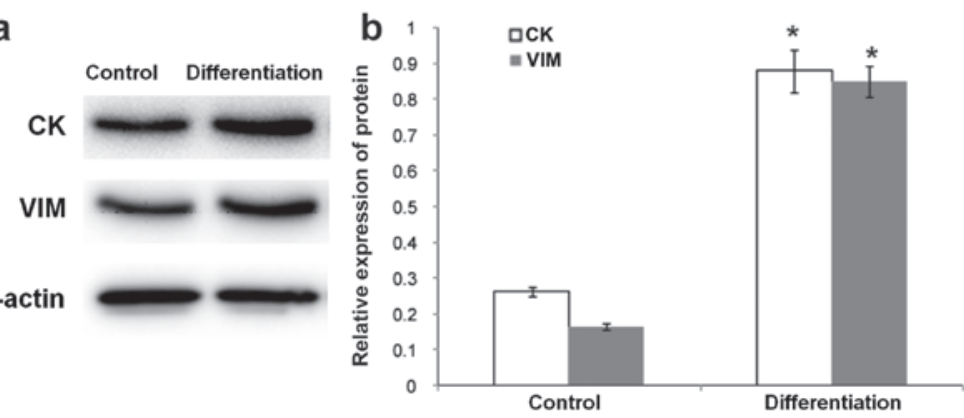

Figure 4. Expression of CK and VIM in cultured normal MenSCs. (A) CK and VIM expression by immunocytochemistry (a and b) prior to and (e and f) following endometrial differentiation using $1 \times 10^{-8} \mathrm{~mol} / 1$ 17ß-E2V and cytokines, (c and d) negative control for (e and f); (g) quantification of expression using a semi-quantitative method. "P $<0.05$ vs. controls; scale bar, $25 \mu \mathrm{m}$. (B) Relative quantification expression of CK and VIM mRNA in controls and MenSCs following endometrial differentiation with $1 \times 10^{-8} \mathrm{~mol} / 17 \beta$-estradiol valerate and cytokines. ${ }^{\prime \prime} \mathrm{P}<0.05$ vs. controls. (C) Relative quantification protein expression of CK and VIM in controls and MenSCs following endometrial differentiation: (a) Western blotting, (b) quantification of CK and VIM expression. CK, cytokeratin; VIM, vimentin; MenSCs, menstrual blood-derived stem cells. 
A

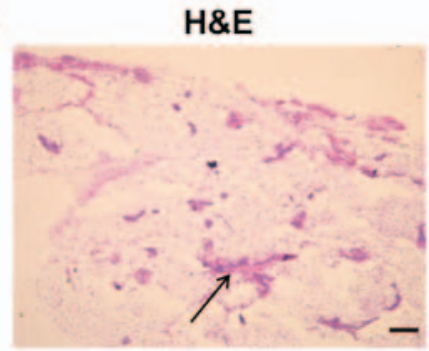

C Negative control

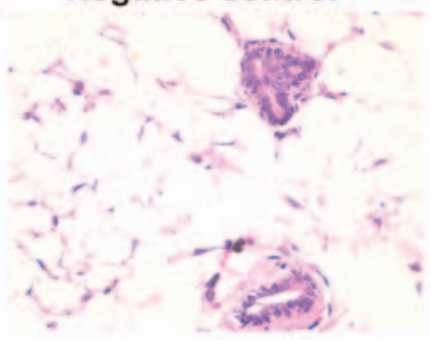

$\mathbf{E}$

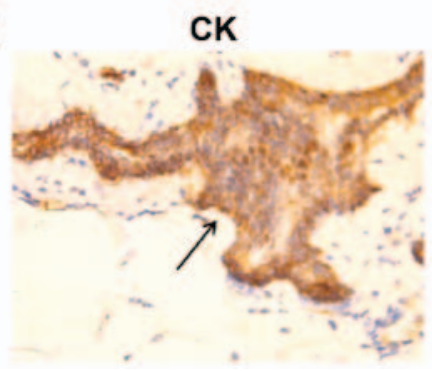

B

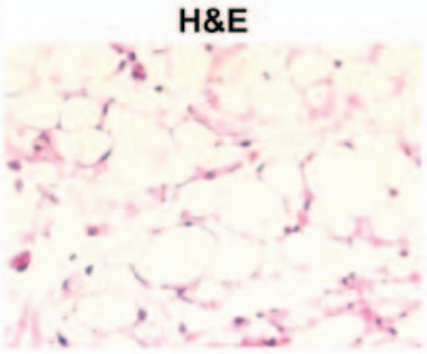

D Negative control

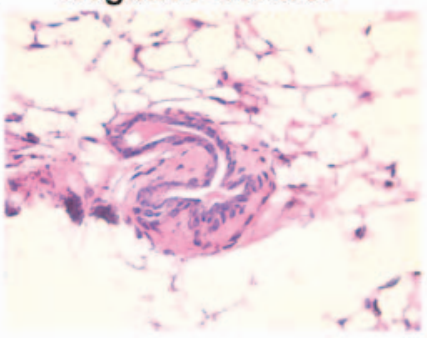

$\mathbf{F}$

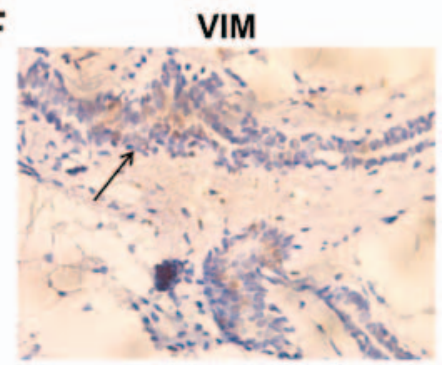

$\mathrm{H}$

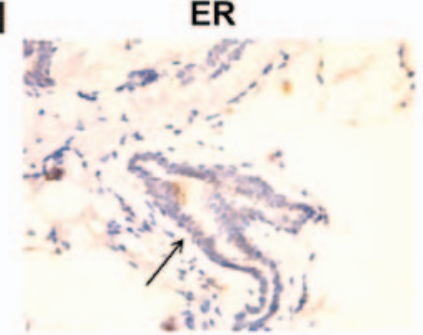

J

ER
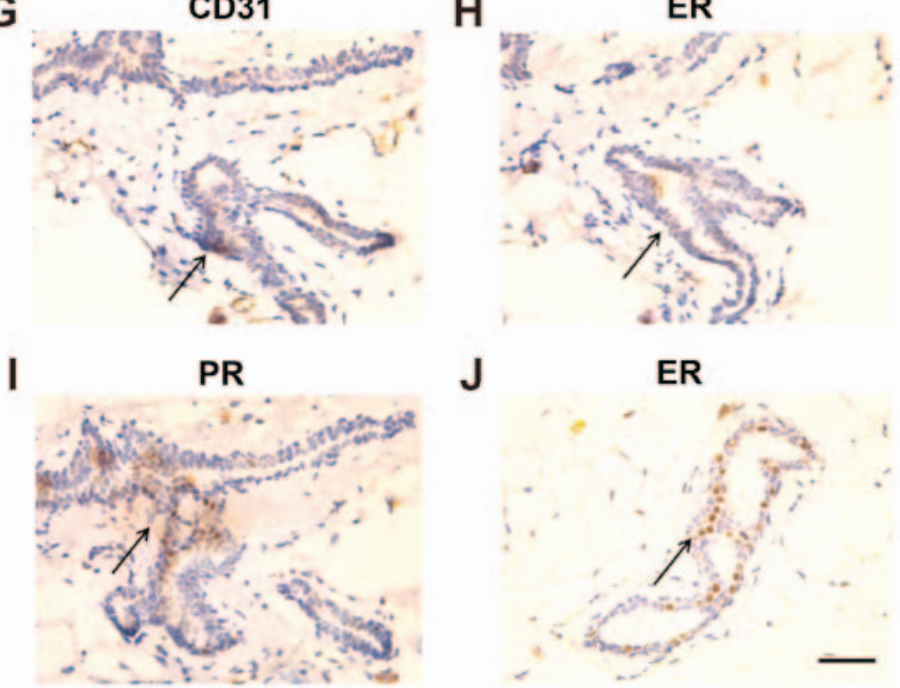

Figure 5. Immunohistochemistry of CK, VIM, CD31, ER and PR in endometrial tissue created by normal MenSCs transplanted in the axillary region of mice (arrows indicate glandular structures). (A) H\&E: Glandular structures scattered in adipose tissue transplanted with MenSCs (scale bar, $200 \mu \mathrm{m}$ ); (B) no glandular structures in adipose tissue transplanted with Dulbecco's modified Eagle's medium. (C and D) negative control for (E). (E) CK: strong positive expression. (F) VIM: moderate intensity expression. (G) CD31: positive expression around blood vessels, but negative in glandular structures. (H) ER: negative following administration of 17ß-E2V; (I) PR: moderate intensity expression following administration of 17ß-E2V; (J) strong positive expression following sequential administration of 17ß-E2V and progesterone. Scale bar, $25 \mu \mathrm{m}$. CK, cytokeratin; VIM, vimentin; CD, cluster of differentiation; ER, estrogen receptor; PR,

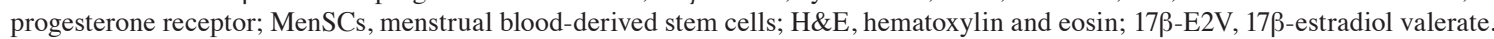

MenSCs differentiate into endometrial tissue in NOD-SCID mice. H\&E staining showed that there were endometrial glandular structures in the adipose tissue following transplantation of MenSCs in mice with the sequential treatment of $17 \beta$-estradiol and progesterone. Certain glands were distorted and others were flat (Fig. 5A), while there was no glandular structures in control (Fig. 5B).

Immunohistochemistry showed that the expression of CK was strongly positive (Fig. 5E). Fig. 5C and D were negative control for Fig. 5E. VIM expression was moderate (Fig. 5F). 
A
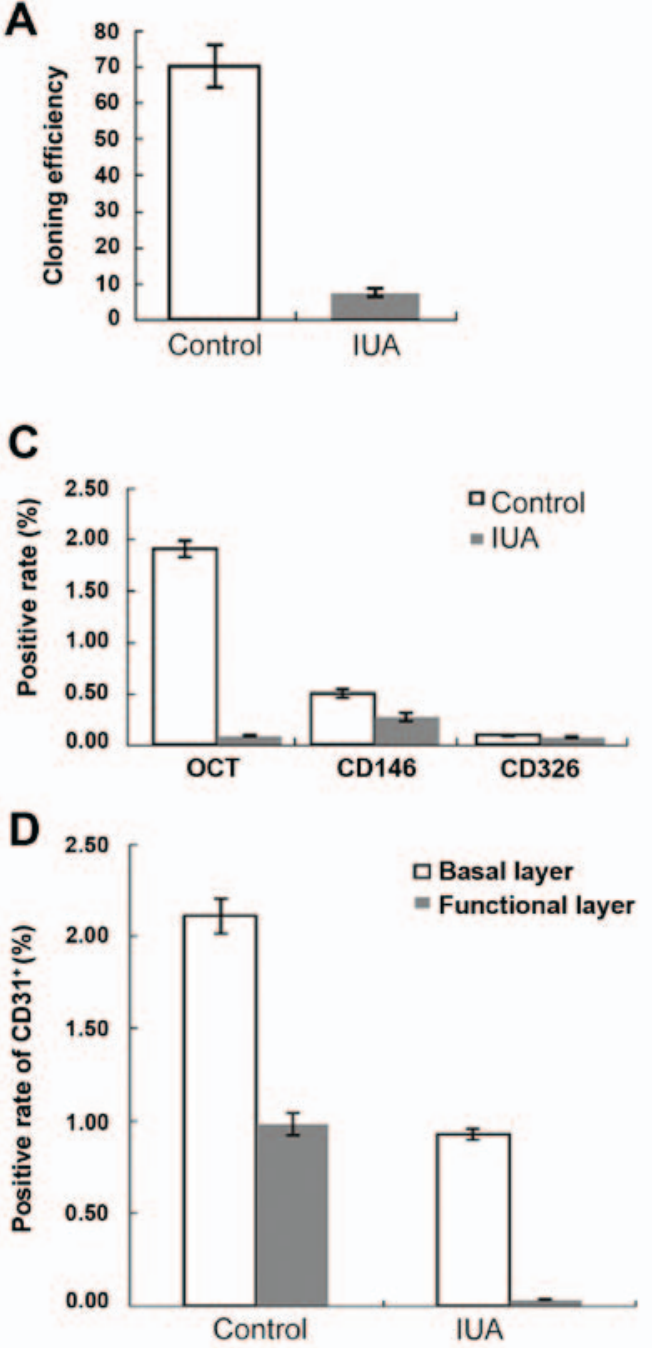

B
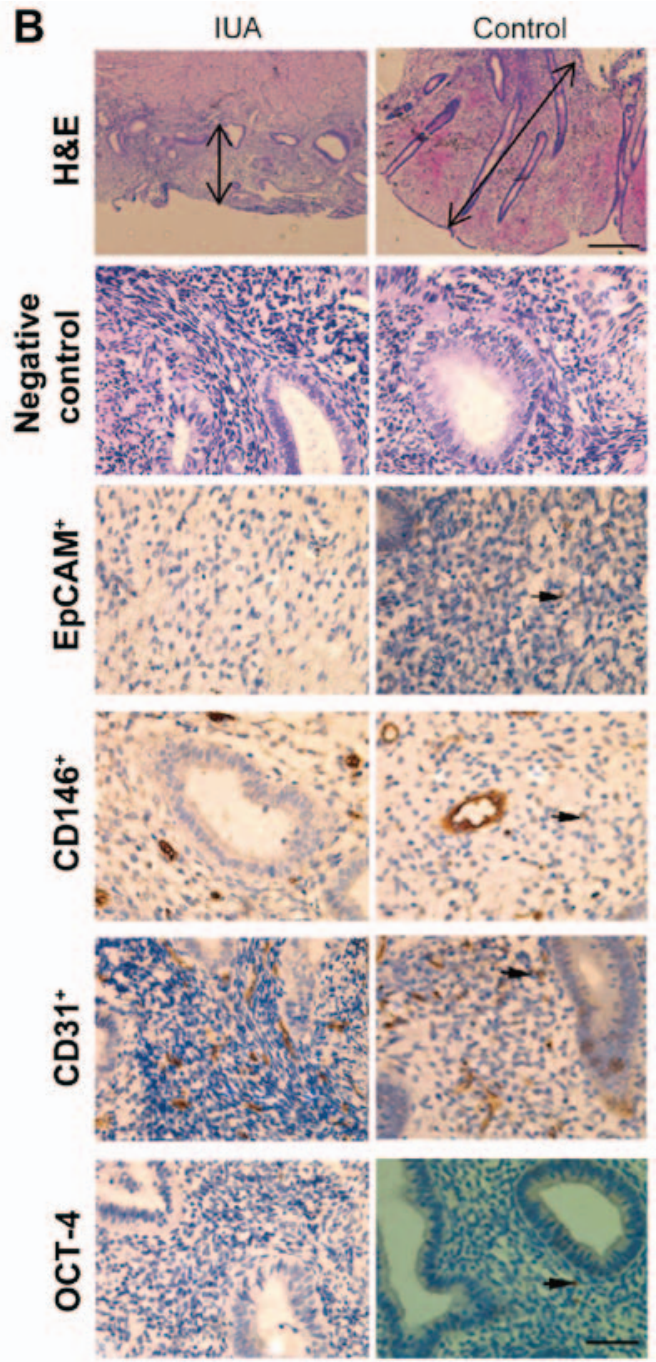

Figure 6. Cloning efficiency and immunohistochemistry of the normal menstrual blood-derived stem cells from healthy women with a normal endometrium and of patients with severe IUA. (A) Cloning efficiency. (B) Staining showing that the endometrium of the IUA group patients was thinner than that in control subjects (arrows); glands and stromal cells are sparse and poorly developed in the IUA group, but densely packed with good development in the control group. Scale bar, $300 \mu \mathrm{m}$. Immunohistochemistry for negative control for OCT-4, EpCAM (CD326), CD146, CD31 and OCT-4. The arrows indicate positive cells. Scale bar, $150 \mu \mathrm{m}$. In these sections, blue dots indicate the cell nucleus staining by hematoxylin. (C) Positivity rates for OCT-4, CD146 and CD326 (EpCAM) in patients with severe IUA and the control. P<0.05 for OCT-4. (D) Immunohistochemistry for CD31 in the basal layer and the functional layer in patients with severe IUA and the control. All P<0.05. IUA, intrauterine adhesion; OCT-4, POU domain class 5 transcription factor 1; HLA-DR, human leukocyte antigen-antigen D related; CD, cluster of differentiation; EpCAM, epithelial cell adhesion molecule.

CD31 expression was positive in the vascular endothelial cells of adipose tissues and negative in the endometrial glandular tissues (Fig. 5G). There was no expression of ER in the induced tissue following the administration of $17 \beta$-estradiol for 4-6 weeks (Fig. 5H), whereas positive expression was determined folloqing sequential treatment of $17 \beta$-estradiol for 4-6 weeks followed by progesterone for 2 weeks (Fig. 5J). The expression of PR was moderately positive after administering $17 \beta$-estradiol for 46 weeks (Fig. 5I).These results suggested that MenSCs could rebuild endometrial tissue in NOD-SCID mice after administering estrogen and progesterone.

Cloning efficiency of MenSCs from patients with severe IUA and healthy women with a normal endometrium. After 15 days of primary culture, there were 340 clones in the control group and 37 clones in the IUA group. The cloning efficiency of the control group was $(6.8 \pm 0.56) \times 10^{-6}$ compared with $(0.74 \pm 0.11) \times 10^{-6}$ for the IUA group $(\mathrm{P}<0.01)$ (Fig. 6A).
Immunohistochemistry of endometrium from patients with severe IUA and normal endometrium in healthy patients. Endometrium samples $\left(5 \mathrm{~mm}^{3}\right)$ were extracted from patients with severe IUA (IUA group, $n=5$ ) (diagnosed by hysteroscopy) and from healthy women with a normal uterine cavity (control group, $\mathrm{n}=5$ ). In the tissues from patients with severe IUA, H\&E staining showed that these tissues were primarily fibrous tissues, with their surface being covered by a thin layer of a small amount of glandular structures, which was suspected to be normal endometrial stroma and glands, but glands and stromal cells were separately located with poor development. In the tissues from the control group, the endometrial layer was thicker than that of patients with IUA, endometrial stromal cells were densely packed and the tissues showed good gland development. In addition, immunohistochemistry showed that OCT-4-positive cells accounted for $\sim 2 \%$ in the control group, whereas the OCT-4-positive cells represented only $<0.1 \%$ of the cells in the IUA group $(\mathrm{P}<0.05)$. 
In the same way, CD146-positive cells accounted for $\sim 1 \%$ in the control group compared with $<0.5 \%$ in the IUA group $(\mathrm{P}<0.05)$. CD326-positive cells represented $<0.1 \%$ of cells in the two groups. Finally, in the controls, CD31-positive cells represented $2 \%$ of the cells in the basal layer and $1 \%$ of the cells in the functional layer, compared with $1 \%$ and $<0.05 \%$, respectively, in the IUA group $(\mathrm{P}<0.05)$ (Fig. 6B-D).

\section{Discussion}

The present study aimed to provide a basis for MenSC use in the treatment of severe IUA. MenSCs were cultured, identified and induced into endometrial cells, and their potential in creating endometrial tissues was assessed in mice. In addition, MenSCs from women with severe IUA were compared with those from healthy women. Immunocy tochemistry and flow cytometry analysis demonstrated that the majority of MenSCs were OCT-4-positive stem cells. Following differentiation, immunocytochemistry, RT-qPCR and western blot analysis showed that the expression of CK and VIM was increased in the MenSCs compared with that in the controls. When transplanted in mice followed by hormonal treatment (estrogen and progesterone), CK, VIM, PR and ER were expressed in the transplantation regions, suggesting that MenSCs could rebuild the endometrium in vivo. The cloning efficiency and OCT-4 positivity of MenSCs from patients with severe IUA was significantly decreased compared with that of controls.

Compared with other tissues, the endometrium can renew and reconstruct itself following/during every menstrual cycle. Therefore, it was inferred that there was a certain type of EnSC in the endometrium that was associated with the self-renewal and reconstruction of the endometrium and angiogenesis during the menstrual cycle (37). EnSCs could be obtained by scraping the endometrium. However, directly scraping the endometrium is an invasive process, and it was suggested that EnSCs could flow out with menstrual blood, namely MenSCs (13). MenSCs have the advantages of being abundantly available after every 28 days, their collection is non-damaging to donors and they have a low cost. As the MenSCs are a human waste product, there is no ethical controversy. Furthermore, MenSCs have a rapid proliferation speed and multi-lineage differentiation potential (13). However, few studies are available about MenSCs.

The present study collected the menstrual blood of healthy reproductive women. MenSCs were separated from the menstrual blood by Ficoll and cultured. During culture, MenSCs were found to grow better in low-glucose medium than in high-glucose DMEM medium, which is usually used (13). Subsequent to culturing, adherent cells proliferated and formed clones, suggesting that MenSCs exhibited the characteristics of stem cells. The growth curve showed that the latency of MenSCs was 7 days, which is supported by a previous study (38). Following passaging, the latency was 3 days and the doubling time was $\sim 36 \mathrm{~h}$, which was shorter than that of mesenchymal stem cells $(13,38)$.

OCT-4 was highly expressed in MenSCs (95.1 $\pm 0.8 \%)$, whereas the expression of STRO-1, CD45 and HLA-DR was very weak. OCT-4, which locates in the nucleus and cytoplasm, is a transcription factor. OCT-4-positive cells are present in pluripotent stem cells (location in nucleus) and multipotent stem cells (location in cytoplasm), and is representative of embryonic-like stem cells (39). All OCT-4-positive cells had the ability to differentiate into three germ layers, but resulted in small teratoma formation with subsequent epigenetic differences between OCT-4-positive and embryonic stem cells $(40,41)$, which may be a reason for a preference of these cells over embryonic stem cells as a regenerative medicine.

One of the most important obstacles in organ transplantation and cellular therapy is immune rejection, and the expression of major histocompatibility complex class II (MHC-II) is associated with the immunogenicity of the cells (31). HLA-DR is a member of the MHC-II family. The present results showed that the expression of HLA-DR is weak in MenSCs, suggesting that MenSCs are unlikely to be rejected following transplantation but with high proliferative capacity.

Rossignoli et al (32) induced MenSCs to differentiate into bone, cartilage and fat cells, suggesting their pluripotency potential. In the present study, PDGF, TGF- $\beta$, EGF and $17 \beta$-estradiol were used to induce MenSCs to differentiate into endometrium in vitro. Under physiological conditions, the endometrium periodically regenerates and exfoliates under the influence of estrogen and progesterone. Estrogen is important to the proliferation and differentiation of the endometrium (42). Results showed that MenSCs could differentiate into epithelial and stromatic cells. MenSCs can secrete the trophic factors for the growth of epithelial cells (13). Following differentiation, CK was selected as a marker for endometrial epithelial cells and VIM for endometrial stromal cells. These results showed that the expression of CK and VIM was high in the differentiation group, suggesting that MenSCs could differentiate into endometrial cells under the influence of estradiol and growth factors.

SCID-consistent mice are T- and B-lymphocyte-deficient mice. Rejection of allograft tissue transplanted in SCID mice is rare. Therefore, this model is ideal for organ transplantation and regeneration. In the present study, the cultured MenSCs were transplanted into the axillary subcutaneous region of NOD-SCID mice, and the mice were sequentially treated with $17 \beta$-estradiol and progesterone. There were endometrial tissues in the transplanted area after 4-6 weeks of administration of $17 \beta$-estradiol and after 2 weeks of administration of progesterone, as indicated by H\&E staining and immunohistochemistry. In H\&E staining, there were numerous endometrial gland structure tissues scattered in the transplanted area in the adipose tissue, while there was none in the control. Upon immunohistochemical analysis, rebuilt endometrial tissue expressed endometrium-related molecules, including CK, VIM, ER and PR, but not CD31, which indicated that rebuilt endometrial tissues were supplied with the nutrients and oxygen through the blood capillaries of surrounding adipose tissues. This is supported by Cervelló et al (43), but is not in accordance with the results found by Masuda et al (44), where CD31 positivity was shown in the reconstructed human endometrium in NOD-SCID mice.

Meng et al (13) successfully isolated and cultured MenSCs. However, thus far, to the best of our knowledge, there has been no study on the clone formation rate of MenSCs from patients with IUA. To investigate the damage to the MenSC population caused by IUA, the present study cultured the menstrual blood and endometrial tissue cells of patients with severe IUA. It was found that the clone formation rate of MenSCs and 
endometrial tissue cells (data not shown) in control subjects was much higher than those from patients with severe IUA. In addition, as determined by immunohistochemistry, OCT-4-, CD146-, CD326- and CD31-positive cells, which presented stem cells or progenitor cells, were significantly decreased in the endometrium of patients with IUA compared with that of healthy women with a normal uterine cavity. All these results suggested that the endometrial stem cells in severe IUA were less potent. However, the study was limited in that it was not designed to assess the cause-to-effect relationship.

In conclusion, MenSCs were able to differentiate into endometrial cells in vitro and reconstructed endometrial tissue in NOD-SCID mice in vivo. Cloning efficiency and OCT-4 expression of MenSCs from patients with IUA were impaired compared with those from healthy women. The present study could provide a theoretical basis for the treatment of IUA with MenSCs. In future studies, MenSCs will be transplanted into the uterus of IUA patients to promote endometrium growth and assist in regaining fertility.

\section{Acknowledgements}

This study was supported by grants from the National Science Fund of China (no. 81370757) and Anhui Medical University (no. 81373671). The authors also acknowledge the assistance provided by Dr Wang Xiaqiu, Dr Zhang Aimei, Dr Fang Wenxiang and Dr Li Chuanying with regard to technical assistance for the experiments.

\section{Competing interests}

The authors declare that they have no competing interests.

\section{References}

1. Evans MJ and Kaufman MH: Establishment in culture of pluripotential cells from mouse embryos. Nature 292: 154-156, 1981.

2. Pittenger MF, Mackay AM, Beck SC, Jaiswal RK, Douglas R, Mosca JD, Moorman MA, Simonetti DW, Craig S and Marshak DR: Multilineage potential of adult human mesenchymal stem cells. Science 284: 143-147, 1999.

3. Arai S, Minjares C, Nagafuchi S and Miyazaki T: Improved experimental procedures for achieving efficient germ line transmission of nonobese diabetic (NOD)-derived embryonic stem cells. Exp Diabesity Res 5: 219-226, 2004.

4. Wolff EF, Gao XB, Yao KV, Andrews ZB, Du H, Elsworth JD and Taylor HS: Endometrial stem cell transplantation restores dopamine production in a Parkinson's disease model. J Cell Mol Med 15: 747-755, 2011.

5. Vaquero J and Zurita M: Bone marrow stromal cells for spinal cord repair: A challenge for contemporary neurobiology. Histol Histopathol 24: 107-116, 2009.

6. Väänänen HK: Mesenchymal stem cells. Ann Med 37: 469-479, 2005.

7. Bobis S, Jarocha D and Majka M: Mesenchymal stem cells: Characteristics and clinical applications. Folia histochemica et cytobiologica/Polish Academy of Sciences. Pol Histochem Cytochemical Soc 44: 215-230, 2006.

8. Yagi H, Soto-Gutierrez A, Parekkadan B, Kitagawa Y, Tompkins RG, Kobayashi N and Yarmush ML: Mesenchymal stem cells: Mechanisms of immunomodulation and homing. Cell Transplant 19: 667-679, 2010.

9. Edwards RG: Stem cells today: B1. Bone marrow stem cells. Reprod Biomed Online 9: 541-583, 2004.

10. Harris DT, Badowski M, Ahmad N and Gaballa MA: The potential of cord blood stem cells for use in regenerative medicine. Expert Opin Biol Ther 7: 1311-1322, 2007.
11. Parker AM and Katz AJ: Adipose-derived stem cells for the regeneration of damaged tissues. Expert Opin Biol Ther 6: 567-578, 2006

12. De Coppi P, Bartsch G Jr, Siddiqui MM, Xu T, Santos CC, Perin L, Mostoslavsky G, Serre AC, Snyder EY, Yoo JJ, et al: Isolation of amniotic stem cell lines with potential for therapy. Nat Biotechnol 25: 100-106, 2007.

13. Meng X, Ichim TE, Zhong J, Rogers A, Yin Z, Jackson J, Wang H, Ge W, Bogin V, Chan KW, et al: Endometrial regenerative cells: A novel stem cell population. J Transl Med 5: 57, 2007.

14. Planka L, Gal P, Kecova H, Klima J, Hlucilova J, Filova E, Amler E, Krupa P, Kren L, Srnec R, et al: Allogeneic and autogenous transplantations of MSCs in treatment of the physeal bone bridge in rabbits. BMC Biotechnol 8: 70, 2008.

15. Dashtdar H, Rothan HA, Tay T, Ahmad RE, Ali R, Tay LX, Chong PP, Kamarul T: A preliminary study comparing the use of allogenic chondrogenic pre-differentiated and undifferentiated mesenchymal stem cells for the repair of full thickness articular cartilage defects in rabbits. J Orthop Res 29: 1336-1342, 2011.

16. Gupta PK, Das AK, Chullikana A and Majumdar AS: Mesenchymal stem cells for cartilage repair in osteoarthritis. Stem Cell Res Ther 3: 25, 2012.

17. Bartunek J, Behfar A, Vanderheyden M, Wijns W and Terzic A: Mesenchymal stem cells and cardiac repair: Principles and practice. J Cardiovase Transl Res 1: 115-119, 2008.

18. Bao X, Feng M, Wei J, Han Q, Zhao H, Li G, Zhu Z, Xing H, An Y, Qin C, et al: Transplantation of Flk- $1^{+}$human bone marrow-derived mesenchymal stem cells promotes angiogenesis and neurogenesis after cerebral ischemia in rats. Eur J Neurosci 34: 87-98, 2011.

19. Zhao Y, Yang C, Wang H, Li H, Du J, Gu W and Jiang J: Therapeutic effects of bone marrow-derived mesenchymal stem cells on pulmonary impact injury complicated with endotoxemia in rats. Int Immunopharmacol 15: 246-253, 2013.

20. Jin SZ, Liu BR, Xu J, Gao FL, Hu ZJ, Wang XH, Pei FH, Hong Y, Hu HY and Han MZ: Ex vivo-expanded bone marrow stem cells home to the liver and ameliorate functional recovery in a mouse model of acute hepatic injury. Hepatobiliary Pancreat Dis Int 11: 66-73, 2012.

21. Fierro FA, Kalomoiris S, Sondergaard CS and Nolta JA: Effects on proliferation and differentiation of multipotent bone marrow stromal cells engineered to express growth factors for combined cell and gene therapy. Stem Cells 29: 1727-1737, 2011.

22. Zhuge Y, Liu Z and Pincus DJ: Improved number and function of bone marrow mesenchymal stem cells by topical estrogen in diabetic murine wound healing. J Surg Res 158: 361-361, 2010.

23. Lédée-Bataille N, Olivennes F, Lefaix JL, Chaouat G, Frydman R and Delanian S: Combined treatment by pentoxifylline and tocopherol for recipient women with a thin endometrium enrolled in an oocyte donation programme. Hum Reprod 17: 1249-1253, 2002.

24. Yu D, Wong YM, Cheong Y, Xia E and Li TC: Asherman syndrome - one century later. Fertil Steril 89: 759-779, 2008.

25. Tu CH, Yang XL, Qin XY, Cai LP and Zhang P: Management of intrauterine adhesions: A novel intrauterine device. Med Hypotheses 81: 394-396, 2013.

26. Zhao J, Zhang Q, Wang Y and Li Y: Uterine infusion with bone marrow mesenchymal stem cells improves endometrium thickness in a rat model of thin endometrium. Reprod Sci 22: 181-188, 2015.

27. Ding L, Li X, Sun H, Su J, Lin N, Péault B, Song T, Yang J, Dai J and Hu Y: Transplantation of bone marrow mesenchymal stem cells on collagen scaffolds for the functional regeneration of injured rat uterus. Biomaterials 35: 4888-4900, 2014.

28. Wang J, Ju B, Pan C, Gu Y, Zhang Y, Sun L, Zhang B and Zhang Y: Application of bone marrow-derived mesenchymal stem cells in the treatment of intrauterine adhesions in rats. Cell Physiol Biochem 39: 1553-1560, 2016.

29. Santamaria X, Cabanillas S, Cervelló I, Arbona C, Raga F, Ferro J, Palmero J, Remohí J, Pellicer A and Simón C: Autologous cell therapy with $\mathrm{CD} 133^{+}$bone marrow-derived stem cells for refractory Asherman's syndrome and endometrial atrophy: A pilot cohort study. Hum Reprod 31: 1087-1096, 2016.

30. Nagori CB, Panchal SY and Patel H: Endometrial regeneration using autologous adult stem cells followed by conception by in vitro fertilization in a patient of severe Asherman's syndrome. J Hum Reprod Sci 4: 43-48, 2011.

31. Zhang MJ, Liu B, Xia W, Sun ZY and Lu KH: Could cells from menstrual blood be a new source for cell-based therapies? Med Hypotheses 72: 252-254, 2009. 
32. Rossignoli F, Caselli A, Grisendi G, Piccinno S, Burns JS, Murgia A, Veronesi E, Loschi P, Masini C, Conte P et al: Isolation, characterization, and transduction of endometrial decidual tissue multipotent mesenchymal stromal/stem cells from menstrual blood. Biomed Res Int 2013: 901821, 2013.

33. Alcayaga-Miranda F, Cuenca J, Luz-Crawford P, Aguila-Díaz C Fernandez A, Figueroa FE and Khoury M: Characterization of menstrual stem cells: Angiogenic effect, migration and hematopoietic stem cell support in comparison with bone marrow mesenchymal stem cells. Stem Cell Res Ther 6: 32, 2015.

34. Ding DC, Chu TY, Chiou SH and Liu HW: Enhanced differentiation and clonogenicity of human endometrial polyp stem cells. Differentiation 81: 172-180, 2011.

35. Zhang WB, Cheng MJ, Huang YT, Jiang W, Cong Q, Zheng YF and $\mathrm{Xu} C \mathrm{CJ}$ : A study in vitro on differentiation of bone marrow mesenchymal stem cells into endometrial epithelial cells in mice. Eur J Obstet Gynecol Reprod Biol 160: 185-190, 2012.

36. Livak and Schmittgen: Analysis of relative gene expression data using real-time quantitative PCR and the $2-\Delta \Delta \mathrm{Ct}$ method. Methods 25: 402-408, 2001.

37. Edwards SL, Werkmeister JA, Rosamilia A, Ramshaw JA, White JF and Gargett CE: Characterisation of clinical and newly fabricated meshes for pelvic organ prolapse repair. J Mech Behav Biomed Mater 23: 53-61, 2013.

38. Tsuji S, Yoshimoto M, Takahashi K, Noda Y, Nakahata T and Heike T: Side population cells contribute to the genesis of human endometrium. Fertil Steril 90 (Suppl 4): 1528-1537, 2008.

39. Gunjal P, Bhartiya D, Metkari S, Manjramkar D and Patel H: Very small embryonic-like stem cells are the elusive mouse endometrial stem cells - a pilot study. J Ovarian Res 8: 9, 2015.
40. Golestaneh N, Kokkinaki M, Pant D, Jiang J, DeStefano D, Fernandez-Bueno C, Rone JD, Haddad BR, Gallicano GI and Dym M: Pluripotent stem cells derived from adult human testes. Stem Cells Dev 18: 1115-1126, 2009.

41. Ratajczak MZ, Shin DM, Ratajczak J, Kucia M and Bartke A: A novel insight into aging: Are there pluripotent very small embryonic-like stem cells (VSELs) in adult tissues overtime depleted in an Igf-1-dependent manner? Aging (Albany NY) 2: 875-883, 2010.

42. Zhang X, Hirai M, Cantero S, Ciubotariu R, Dobrila L, Hirsh A, Igura K, Satoh H, Yokomi I, Nishimura T, et al: Isolation and characterization of mesenchymal stem cells from human umbilical cord blood: Reevaluation of critical factors for successful isolation and high ability to proliferate and differentiate to chondrocytes as compared to mesenchymal stem cells from bone marrow and adipose tissue. J Cell Biochem 112: 1206-1218, 2011.

43. Cervelló I, Mas A, Gil-Sanchis C, Peris L, Faus A, Saunders PT, Critchley HO and Simón C: Reconstruction of endometrium from human endometrial side population cell lines. PLoS One 6: e21221, 2011

44. Masuda H, Maruyama T, Hiratsu E, Yamane J, Iwanami A, Nagashima T, Ono M, Miyoshi H, Okano HJ, Ito M, et al: Noninvasive and real-time assessment of reconstructed functional human endometrium in NOD/SCID/gamma c(null) immunodeficient mice. Proc Natl Acad Sci USA 104: 1925-1930, 2007. 